\title{
Impact fatigue damage of coated glass fibre reinforced polymer laminate
}

Fraisse, Anthony; Bech, Jakob Ilsted; Borum, Kaj Kvisgaard; Fedorov, Vladimir; Johansen, Nicolai Frost-Jensen; McGugan, Malcolm; Mishnaevsky, Leon; Kusano, Yukihiro

Published in:

Renewable Energy

Link to article, DOI:

10.1016/j.renene.2018.04.043

Publication date:

2018

Document Version

Peer reviewed version

Link back to DTU Orbit

Citation (APA):

Fraisse, A., Bech, J. I., Borum, K. K., Fedorov, V., Johansen, N. F-J., McGugan, M., Mishnaevsky, L., \& Kusano, Y. (2018). Impact fatigue damage of coated glass fibre reinforced polymer laminate. Renewable Energy, 11021112. https://doi.org/10.1016/j.renene.2018.04.043

\section{General rights}

Copyright and moral rights for the publications made accessible in the public portal are retained by the authors and/or other copyright owners and it is a condition of accessing publications that users recognise and abide by the legal requirements associated with these rights.

- Users may download and print one copy of any publication from the public portal for the purpose of private study or research.

- You may not further distribute the material or use it for any profit-making activity or commercial gain

- You may freely distribute the URL identifying the publication in the public portal 
1 Impact fatigue damage of coated glass fibre reinforced polymer laminate

\author{
Anthony Fraisse ${ }^{1}$, Jakob Ilsted Bech ${ }^{1}$, Kaj Kvisgaard Borum ${ }^{1}$, Vladimir Fedorov ${ }^{1}$, Nicolai Frost-Jensen \\ Johansen $^{2}$, Malcolm McGugan ${ }^{1}$, Leon Mishnaevsky Jr. ${ }^{1}$, Yukihiro Kusano ${ }^{1}$ \\ ${ }^{1}$ Department of Wind Energy, Technical University of Denmark, Ris $\emptyset$ Campus, 4000 Roskilde, Denmark \\ ${ }^{2}$ Department of Mechanical Engineering, Technical University of Denmark, Lyngby Campus, 2800 Lyngby, \\ Denmark
}

\begin{abstract}
Impact fatigue caused by rain droplets, also called rain erosion, is a severe problem for wind turbine blades and aircraft. In this work, an assessment of impact fatigue on a glass fibre reinforced polymer laminate with a gelcoat is presented and the damage mechanisms are investigated. A single point impact fatigue tester is developed to generate impact fatigue damage and SN data. Rubber balls are repeatedly impacted on a single location of the coated laminate. Each impact induces transient stresses in the coated laminate. After repeated impacts, these stresses generate cracks, leading to the removal of the coating and damage to the laminate. High-resolution digital imaging is used to determine the incubation time until the onset of coating damage, and generate an SN curve. An acoustic emission sensor placed at the back of the laminate monitors changes in acoustic response as damage develops in the coated laminate. The subsurface cracks are studied and mapped by 3D X-ray computed tomography. A finite element method model of the impact shows the impact stresses in the coating and the laminate. The stresses seen in the model are compared to cracks found by 3D tomography. The damage is also evaluated by ultrasonic scanning.
\end{abstract}

Keywords: Impact fatigue; glass fibre reinforced polymer; leading edge erosion; acoustic emission; X-ray computed tomography; ultrasound scanning

\title{
1. Introduction
}

Wind energy is recognized as a key renewable energy source, reducing dependency on fossil fuels [ 1, 2, 3, 4]. There are a variety of designs for energy generation by wind, but in all cases, the kinetic energy of wind is converted to electrical energy. The three bladed horizontal axis wind turbine is a common design, comprising rotor blades, a tower and a power converting part including a generator and a gear box. Since the power generation capacity of a wind turbine highly depends on the swept area of the blades, lighter and larger blades are demanded $[5,6]$. Fibre reinforced polymer composite materials can meet the demand for lighter and larger wind turbine blades due to their high strength-to-mass ratio, a high stiffness-to-mass ratio, good fatigue resistance, corrosion resistance, flexible formability and low thermal expansion.

Surfaces of wind turbine blades in both onshore- and offshore-installations are exposed environmental and tribological effects over their operational lifetimes [7], including extreme wind/gusts, rain showers, hailstone showers, airborne particles of sand, snow, icing, extreme temperatures and ultraviolet light exposure. Among them rain erosion is often thought to be a major damage source [8]. In particular, the leading edge of the blade tips, whose speed is commonly greater than $80 \mathrm{~m} \mathrm{~s}^{-1}$ [9], can experience significant damage, and thus a protective coating is usually applied. Such damages are collectively called "leading edge erosion". However, erosion is only one of the damage phenomena, and in fact, very little is known about the different damage 
modes and mechanisms. All wind farms require frequent visual inspection of the blades and subsequent repair or replacement due to unpredictable damages.

Attempts to reproduce the rain erosion damage phenomena in laboratory scale can be found in literature eg [10]. Rain erosion testing (RET) is used to experimentally assess rain erosion performances. The most common test for rain erosion in wind energy applications is the rotating arm rig, where a rotor is rotating in a rain field of generated droplets [11]. It was originally developed for aerospace materials. Despite the prevalence of this test method there is a problem in reproducibility when different test setups are compared. This is mainly due to turbulence in the test rigs, different droplet size distributions, and other parameters which are difficult to control. In addition, the evaluation mainly relies on visual inspection of the protective coatings to document damage and delamination, and a measurement of the material mass loss, without taking account of damage modes. The testing requires these operation to be stopped and the blade sample unmounted, making observation limited to few discrete intervals, and an observation of exact failure times and mechanisms difficult. Considering these circumstances, development of a highly controlled impact test method, in-situ monitoring and damage characterisation techniques are needed for studying the impact damage mechanisms in detail. Impact damage and impact fatigue are also studied by other means like drop weight tests and shooting with projectiles [12].

The effect of repeated mechanical loading in a material's lifetime has been studied based on a concept of cumulative fatigue damages $[13,14]$. This concept can be applied to the impact fatigue damage of coated systems. There is usually an initial incubation period during which damage accumulates in the material while no visible damage and no functional loss is detected, as shown in Figure 1. After the incubation period, a steady damage evolution may be measured. For the leading edge erosion of wind turbine blades, a delta mass method is commonly used, in which mass loss of a material is measured at discrete time intervals. With this method, the impact damage process may be simplified to two discrete stages. In the incubation period, small cracks begin to form inside the material and each crack further extends as the impacts continue. At some point, the cumulative damage results in cracks merging and an initial removal of a portion of the material at the surface, followed by a period of steady mass loss. Drawbacks of the delta mass methods are that in-situ measurements are difficult to perform due to severe mechanical impacts and the fact that initial damages including crack creation are not detected. Therefore, it is desirable to use enhanced inspection techniques suitable for in-situ observation and monitoring, such as visual imaging and acoustic detection.

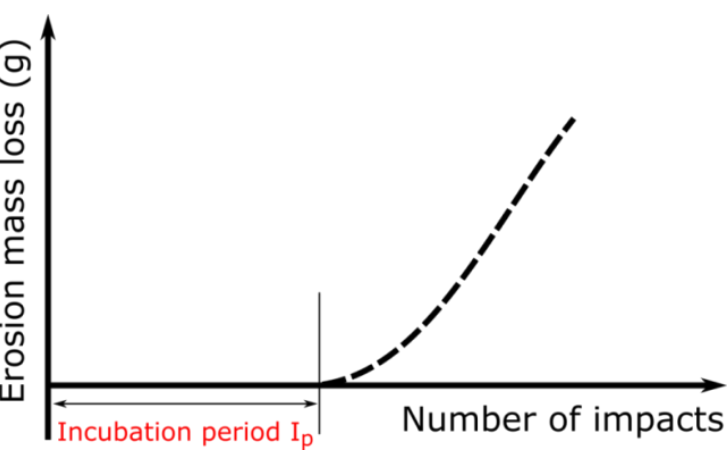

Figure 1. Schematic diagram of erosion mass loss as a function of the number of impacts, accompanying an incubation period $\mathrm{I}_{\mathrm{p}}$ at the initial stage of impacts.

In the present work, a newly developed single point impact fatigue test (SPIFT) is presented. Ex-situ measurements of ultrasonic scanning and X-ray tomography were performed to identify initial defects in specimens. After that, the impact fatigue test was carried out. Rubber balls are used to impact specimen surfaces with a defined impact-speed and interval. During the impact test, the specimen surface was observed in-situ by digital imaging, and acoustic signals were measured. After the impacts, the specimens were again 
characterised by the ex-situ methods for damage analysis. The results were compared with stress wave propagation in the specimen simulated by a finite element method (FEM) model.

\section{Experimental setup and methods}

\subsection{Materials}

Flat glass fibre reinforced polymer (GFRP) laminates coated with an epoxy based gelcoat were manufactured by vacuum infusion as shown in Figure 3. The uncured gelcoat layer (Huntman, RenGel, SW 5200 / Ren NY 5212 , density $=1.5 \mathrm{~g} \mathrm{~cm}^{-3}$ ) was first placed on a flat mould surface, on which fibre fabric layers were placed in addition to process aid foils. The symmetric fibre layup was established using twelve fabric layers. The fabric layers in the lay-up were biaxial $\left( \pm 45^{\circ}\right) 444 \mathrm{~g} \mathrm{~m}^{-2}$ layers (Saertex, density $=2.60 \mathrm{~g} \mathrm{~cm}^{-3}$ ). A Huntsman epoxy resin (epoxy: LY 1564 SP, hardener: Aradur 3487. Huntsmann) was infused into the mould to impregnate the fibres. Panels were cured at $40^{\circ} \mathrm{C}$ for $21 \mathrm{~h}$ and post-cured at $80^{\circ} \mathrm{C}$ for $5 \mathrm{~h}$.

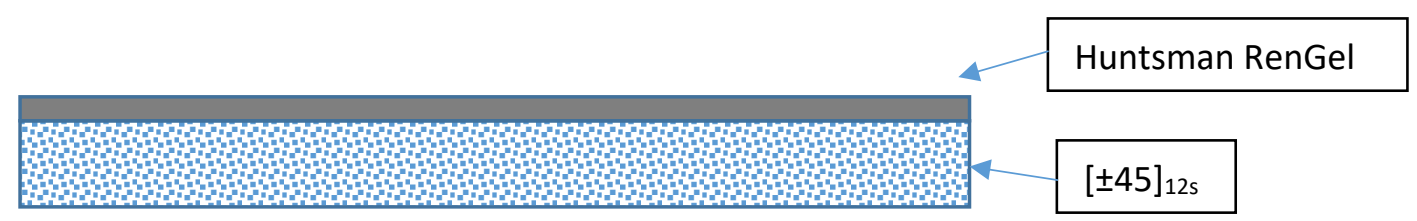

Figure 3. Material lay-up.

\section{2. $\quad$ Projectile material}

The projectiles used for impact testing are $\varnothing 6 \mathrm{~mm}$ balls made from Buna nitrile rubber (NBR60), with a shore hardness of 60 . The mass of each ball is $0.143 \mathrm{~g}$ giving each ball a density of $1.267 \mathrm{~kg} \mathrm{~m}^{-3}$. The choice of NBR60 for the projectiles is based on the need for a low-modulus material that would remain intact during repeated impacts, while still having a density close to that of water. In order to reduce friction between the barrel and the rubber ball while firing, a small amount of silicon oil lubrication was used to coat the rubber balls. Figure 4 shows the projectile deforming upon impact with the target. Using high-speed imaging to visualise this deformation, it can be seen that the degree of deformation is dependent upon impact speed, ranging from $\varnothing 6.9 \mathrm{~mm}$ at $88 \mathrm{~m} \mathrm{~s}^{-1}$ to $\varnothing 8 \mathrm{~mm}$ at $167 \mathrm{~m} \mathrm{~s}^{-1}$.

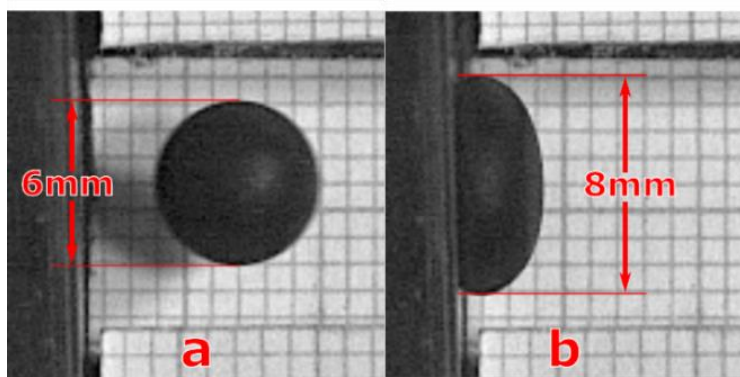

Figure 4. High-speed imaging of the $6 \mathrm{~mm}$ rubber balls captured using a Phantom v2512-fast high-speed camera at $380116 \mathrm{fps} 2.1 \mu \mathrm{s}$ exposure time. (a) just before impact travelling at $167.02 \mathrm{~m} \mathrm{~s}^{-1}$. (b) the deformed projectile at $\emptyset 8 \mathrm{~mm}, 26.15 \mu \mathrm{s}$ after the initial impact. 
The SPIFT repeatedly shoots rubber balls at a coated $15 \times 40 \times 5 \mathrm{~mm}^{3}$ laminate. The shooter is a Valken V12 electro-pneumatic firing engine, driven by compressed air and containing a plunger and two magnetic valves. The schematic setup is illustrated in Figure 5. The balls are fed by gravity into the firing mechanism from a vibrating magazine hopper. The plunger lets the balls into the chamber one by one. After entering the chamber the rubber ball is loaded into the barrel, whereupon compressed air is released to accelerate the rubber ball through the barrel. After leaving the barrel the rubber ball passes through an optical speed trap (Airchrony Mk.3) recording the exit velocity of the rubber ball. Having passed the speed trap the rubber ball hits the target. The setup can shoot up to 5 rubber balls per second with velocities up to $170 \mathrm{~m} \mathrm{~s}^{-1}$. The velocity is regulated by the applied air pressure. The time interval between shots and the number of shots in a series are controlled by a programmable microprocessor giving input to the magnetic valves. During testing the impact area is monitored using a digital microscope camera.

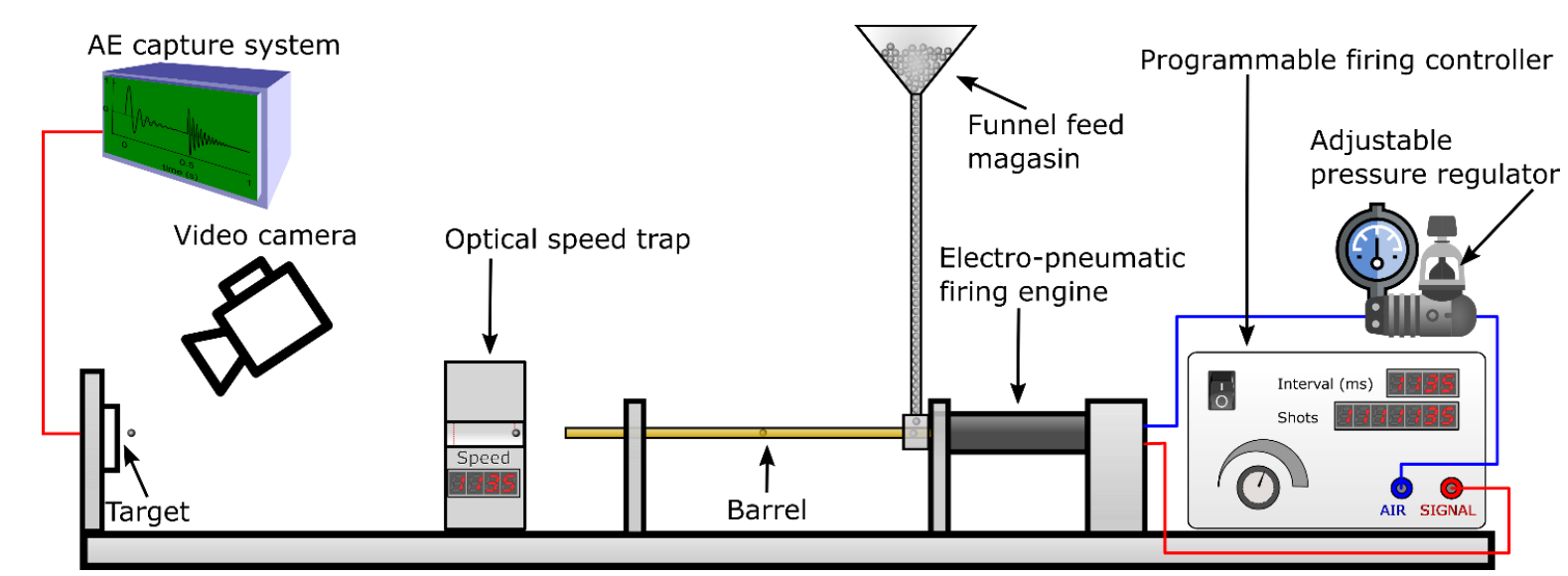

Figure 5. SPIFT setup.

\subsection{Digital video acquisition}

As the primary means of damage detection, high-resolution digital video images were captured at a 3.1 Megapixel $(2048 \times 1534 @ 10 \mathrm{~Hz})$ with an AM7915MZTL long working distance USB microscope from Dinolite. Using a working distance of $120 \mathrm{~mm}$, a $18 \times 13 \mathrm{~mm}^{2}$ field of view is obtained resulting in 13.5 pixels mm 2 .

\section{5 .}

Acoustic emission

Acoustic Emission (AE) refers to the elastic stress wave energy that travels through a material when it cracks, or an impact takes place, or some other process occurs that imparts or releases mechanical energy into the system. That energy then travels through the material and can be detected by surface mounted sensors. When the transient energy generated by an impact (as in this case) reaches a surface-mounted AE sensor, the micro accelerations activate the piezoelectric crystal inside the sensor. The fluctuating charge of the piezoelectric crystal is then amplified and the resulting waveform is delivered to the AE system which digitises and processes the information. 


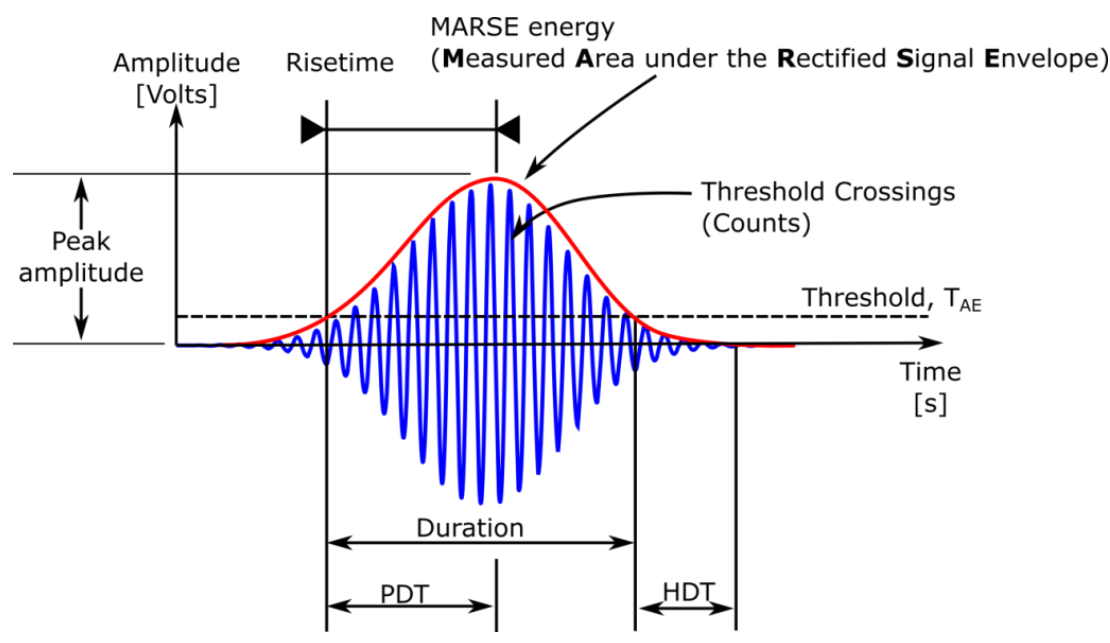

Figure 6. Amplified waveform schematically showing timing parameters and extracted AE features.

In order to extract relevant features from the transient waveform, user defined detection and timing parameters must be provided as illustrated in Figure 6. These include a threshold voltage $\left(\mathrm{T}_{\mathrm{AE}}\right)$ above which an AE "hit" will be registered, a Peak definition time (PDT) specifying the time allowed after the detection of a "hit" to determine the peak value for that hit - if PDT is too long then false peak values are likely to be recorded. If too short, the true value may not be identified. Other timing parameters like hit definition time (HDT) and hit lockout time (HLT) must also be specified and can likewise result in incorrect waveform characterization if set incorrectly.

With an AE sensor on the SPIFT specimen we will register the stress wave response resulting from each individual impact event; an impact detection. In addition we hope, by comparing changes in the extracted AE waveform features, to show an impact characterisation that will discriminate between different velocities and different laminate material, specimen dimensions and attachment/mountings, different coating/protection layers, and ultimately the damage state (of both coating and laminate).

Figure 5 in section 2.3 shows the SPIFT setup with a single AE sensor attached to the reverse side of the coated laminate. A laboratory optimised Express- 8 AE board and chassis system from Mistras Group ${ }^{1}$ was used to detect and analyse the signals. As only a small space was available for instrumentation, a miniature Pico sensor ${ }^{2}$ was used.

With the sensor in place and the system running a problem became apparent. Each rubber ball impact resulted in multiple AE "hits" being detected. Altering the user defined detection and timing configuration to the following (Threshold $50 \mathrm{~dB}$, PDT $150 \mu \mathrm{s}$, HDT $1500 \mu$ s and HLT $1500 \mu \mathrm{s}$ ) solved this issue and thereafter the system recorded a unique individual AE event for every impact occurring.

\section{6. $\quad$ Ultrasound scanning}

Ultrasound refers to sound waves, traveling through a material with a frequency far higher than the audible limit of human hearing. For material evaluation, a typical frequency range is defined between 1 and $50 \mathrm{MHz}$. For high resolution measurements, the test was conducted in water using a focused transducer.

A focused ultrasonic wave was sent into the material by the transducer and reflections from interfaces were recorded by the same transducer (Figure 7).

\footnotetext{
${ }^{1}$ http://www.physicalacoustics.com/by-product/micro-II-express/

2 http://www.physicalacoustics.com/content/literature/sensors/Model_PICO.pdf
} 
Each solid material and liquid has it's own specific ultrasonic impedance Z. In linear elastic materials, the impedance is a function of the material density $(\rho)$ and the sound speed of sound $c$ in the given material, $Z=$ $\rho \cdot c$. If the ultrasonic wave meets a different material with a different impedance, the ultrasonic wave will be partly reflected. The larger the difference between the impedance of the two materials, the larger the energy reflection. Therefore, voids or cracks perpendicular to the wave are relatively easy to detect using ultrasound due to the large difference in impedance between air and solid material. The scanner probe was mounted on a gantry that moved in X-Y-Z-planes controlled by stepper-motors. The resulting line scans were combined into a $2 \mathrm{D}$ raster image. At each raster point, an ultrasonic wave was sent into the material and the response was recorded. The resulting scan may be presented as a $2 \mathrm{D}$ contour plot showing local properties using a colourscale.

There are three scenarios describing the wave interaction with defects. In the first one (1), the ultrasound waves are reflected directly back from the laminate. Since the surface is normal to the sensor, the intensity of the reflected echo is strong. In scenarios 2 and 3 most of the ultrasonic wave is reflected at an angle which gives a weak response to the sensor. Table 1 summarises the parameters selected for the ultrasound scanning.

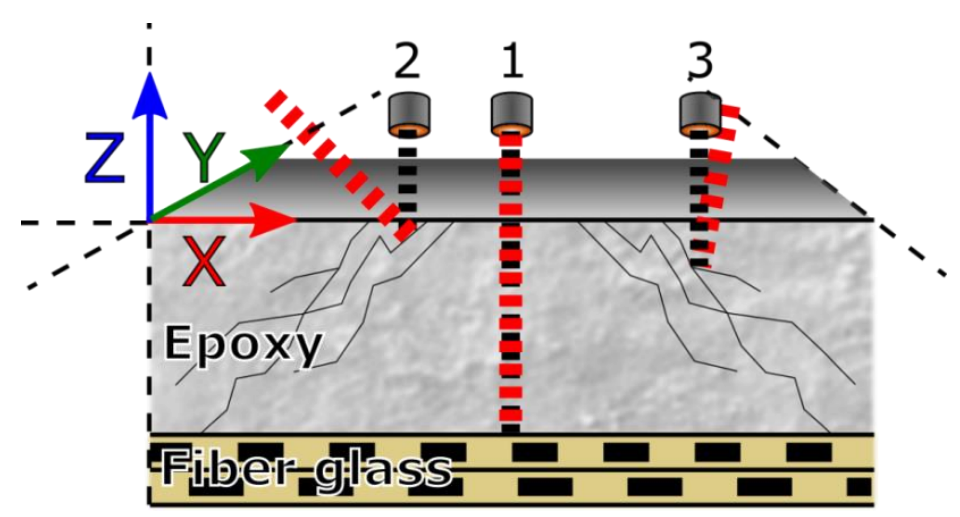

Figure 7. Scenarios of ultrasound interaction with defects. See the main text for further details.

Table 1. Ultrasound parameters.

\begin{tabular}{|c|c|c|}
\hline Transducer frequency & Scan resolution $\mathrm{X}$ and $\mathrm{Y}$ & $\begin{array}{c}\text { Distance from transducer } \\
\text { to sample surface }\end{array}$ \\
\hline $\begin{array}{c}50 \mathrm{MHz} \\
\text { (focused, } 0.2 \mathrm{~mm} \mathrm{6} \mathrm{dB} \mathrm{limit)}\end{array}$ & $\begin{array}{c}0.1 \mathrm{~mm} \text { for full sample, } 0.02 \\
\text { mm for zoom scan }\end{array}$ & $15.3 \mathrm{~mm}$ \\
\hline
\end{tabular}

2.7.

3D X-ray computed tomography

In the X-ray scanner, the specimen is placed between an X-ray source and a detector. The intensity of the radiation reaching the sensor depends on the density of the material. The sensor has a number of "pixels/sensors" each getting charged by the X-ray transmitted through the specimen during the exposure. Thus a $2 \mathrm{D}$ image presenting the variation of damping in the specimen is generated. By gradually turning $180^{\circ}$ and scanning for each few degrees a number of 2D images are generated, each presenting a plane in the scanned volume. Using Computerized tomography a 3D matrix of the damping in each scanned volume is generated. Using imaging software any plane/slice of the scanned volume can be carefully evaluated. The software permits both an investigation of each of the 2D planes from the 3 directions, as well as presenting the overall $3 \mathrm{D}$ volume.

The X-ray scans were performed using a Zeiss Xradia 520 Versa. This device can scan a specimen with a characteristic size up to $10 \mathrm{~cm}$ with a resolution $100 \mu \mathrm{m}$ and smaller samples with a resolution down to less 
than $1 \mu \mathrm{m}$. In this study, the sample size was defined to be $15 \mathrm{~mm}$ wide and $40 \mathrm{~mm}$ long. This geometry has been defined in order to have a representative volume of the material to test and to ensure a good scan quality. 3D ex-situ tomography was chosen as the characterisation technique for its nondestructive aspect and capacity to identify and follow the damage mechanisms in the coated laminate during impact fatigue. The repeatability of the method can be a challenge, and therefore special care was taken while mounting the samples in the scanner. As shown in Figure 8, a hole was drilled in the sample in which a pin was inserted to precisely index the specimen for each scan.

The 3D scans shown in this study were obtained using the parameters gathered in Table 2.

Table 2. Scan parameters.

\begin{tabular}{|c|c|c|c|c|c|c|c|c|c|}
\hline Objective & Binning & $\begin{array}{c}\text { Exposure } \\
\text { time (s) }\end{array}$ & Filtre & $\begin{array}{c}\text { Voltage } \\
(\mathrm{kV})\end{array}$ & $\begin{array}{c}\text { Field } \\
\text { of } \\
\text { view } \\
(\mathrm{mm})\end{array}$ & $\begin{array}{c}\text { Distance } \\
\text { to } \\
\text { detector } \\
(\mathrm{mm})\end{array}$ & $\begin{array}{c}\text { Distance } \\
\text { to source } \\
(\mathrm{mm})\end{array}$ & $\begin{array}{c}\text { Scan } \\
\text { rotation } \\
(\mathrm{deg})\end{array}$ & $\begin{array}{c}\text { Number of } \\
\text { projections }\end{array}$ \\
\hline $\mathrm{X} 4$ & 2 & 14 & $\mathrm{LE} 4$ & 55 & 2.04 & 40 & 17 & 360 & 5201 \\
\hline
\end{tabular}

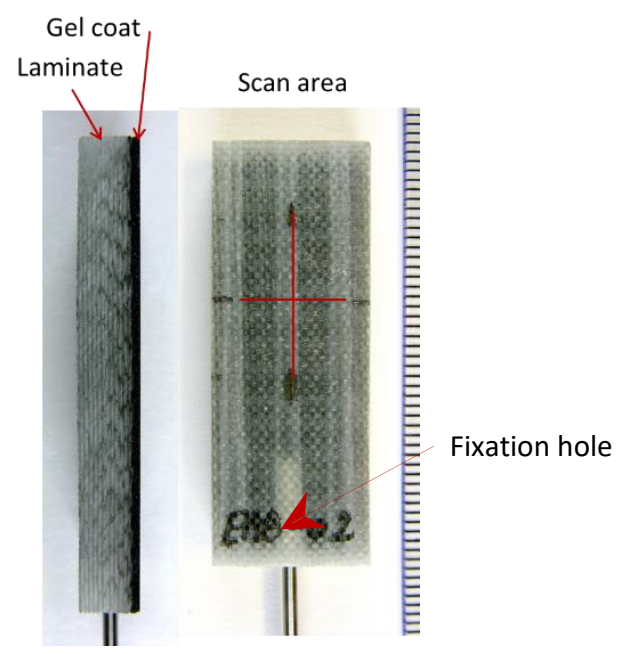

Figure 8 . The coated laminate with mounted pin.

\subsection{FEM model}

An FEM of impact onto the coated laminate was developed to study internal transient stresses. Two models were developed: One for a rubber ball, and another for a water droplet impacting the coated laminate. Since the impact process implies a large particle and local laminate deformations, a special finite element configuration was developed. The target laminate was modelled using the commonly used Eulerian domain, where each finite element corresponds to the specific material particles. The projectiles (rubber ball and water droplet) were specified in the Lagrangian domain, where the material was not fixed to the mesh but flew through it. The finite models were developed in ABAQUS which allows for combinations and interactions of Eulerian and Lagrangian domains in the same model.

The model consists of a target laminated with 12 uni-directional $0.6 \mathrm{~mm}$ thick glass-fibre layers and a $1.1 \mathrm{~mm}$ thick gelcoat layer on top of the laminate, reproducing the laminate used for the experiments (Figure 3). The projectiles are $6 \mathrm{~mm}$ diameter balls approaching the target laminate at a specified velocity. The boundary conditions for the laminate were defined as simply supported surfaces of the laminate in contact with the test 
rig fixture system. This might not be the most accurate representation of the actual boundary conditions, especially for the transient processes, but this method is assumed as reasonable.

For acceleration of the solution process, the entire model domain is symmetric with respect to geometry, loads, and boundary conditions. Therefore, the model was split into two symmetric parts and only one of them was modelled with proper symmetry conditions.

Since the impact transient process is fast with large stress gradients in space and stress variations in time, the explicit method was used to solve the FEM model at each time step. In contrast to the implicit method which is commonly used for slow quasi-static processes, the explicit method does not seek for equilibrium of the system at each time step. This allows significantly faster solutions, with moderate risks for the solution to drift away from the solution obtained with implicit method.

\section{Results and discussion}

\section{1. $\quad$ Failure criterion by visual damage}

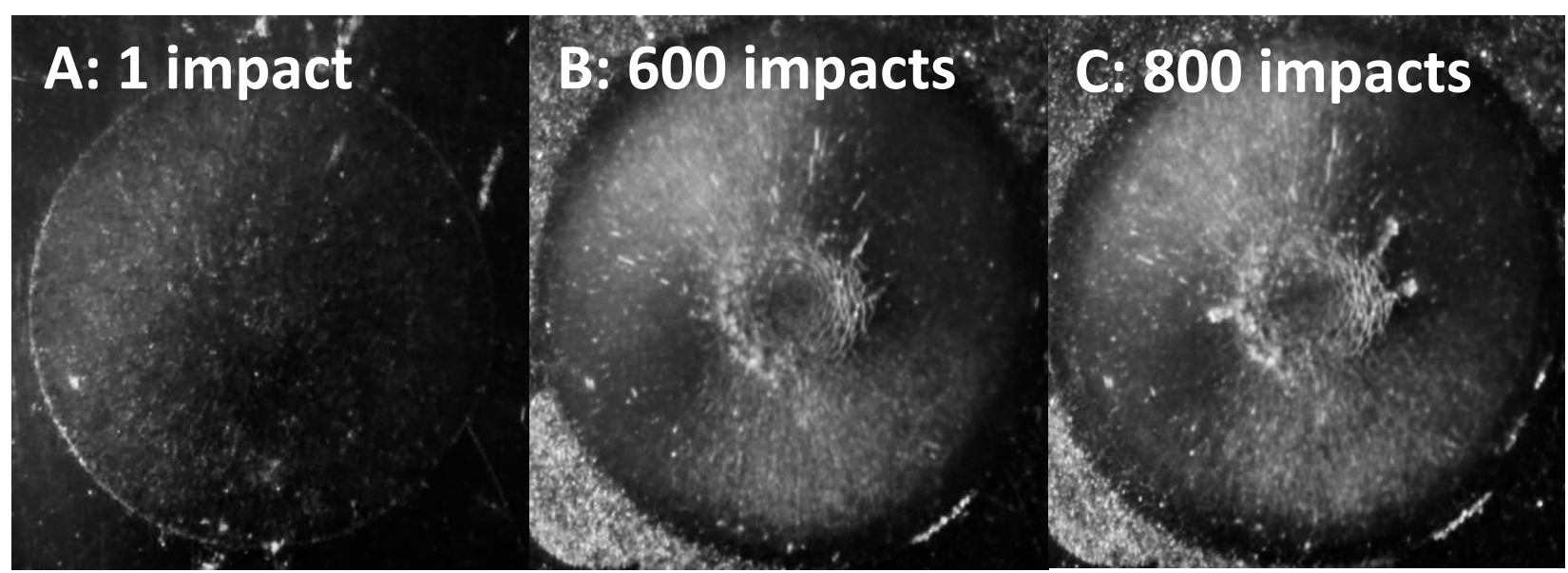

Figure 9. Images from the in-situ video capture showing different stages of testing impact speed $129.8 \pm 0.82$ $\mathrm{m} \mathrm{s}^{-1}$ at impact frequency $2 \mathrm{~Hz}$. A: Impacted but visually undamaged. B: Impacted sample with circular crazing evident in high-stress impact region. C: Impacted sample just after first material loss denoting the end of incubation time.

One of the advantages of SPIFT is that it allows simple in-situ damage assessment. In conventional RET, the rotating target, in a rain and water mist environment, makes the capture of high-resolution images difficult. Therefore, high-resolution imaging is usually performed outside the RET setup.

Since samples used in SPIFT are stationary, high-quality image or video capture is a relatively simple task and is achieved using the camera described in section 2.4.

From the captured visual data the incubation time was identified. End of incubation was determined by the time at which the first material loss is observed denoting the onset of constant erosion (see Figure 9). In Figure 9, the image B exemplifies a sample close to the end of incubation, where a part of the coating is about to be detached from the laminate. Hereafter, the partial removal of the coating is referred to as "damaged".

Depending on the test conditions, the incubation time could be determined within 2-20 impacts. The higher the impact speed, the more abrupt the change from un-damaged to damaged, whereas at lower impact speeds the change was less abrupt. As seen in Figure 9, circular crazing is observed prior to erosion, and is consistent 
across all samples. These circular cracks bear a strong resemblance to literature [15], in which the MIJA (Multiple Impact Jet Apparatus [16] was used to induce erosion in carbon nanotube reinforced polymers. The fact that the crazing starts away from the point of impact is due to peak stress intensity coupled with the specific material properties of both projectile and target, as well as the impact speed.

The crazing is analysed further in section 3.4 and 3.5 where X-ray tomography and FEM modelling are combined to show a correlation between stress waves and crack propagation.

The data pairs (impacts to end of incubation, speed) are plotted in an SN or Wöhler diagram shown in Figure 10 , where traditionally the independent parameter, velocity, is plotted on the vertical axis and the dependent parameter, number of impacts before visible erosion, is plotted on the horizontal axis of a semi log. Such a representation of data is known from fatigue testing of materials [17], where the number of load cycles causing damage is shown as a function of the magnitude of each load cycle (for example stress range). The fatigue data are fitted with a power function as shown in Figure 10.

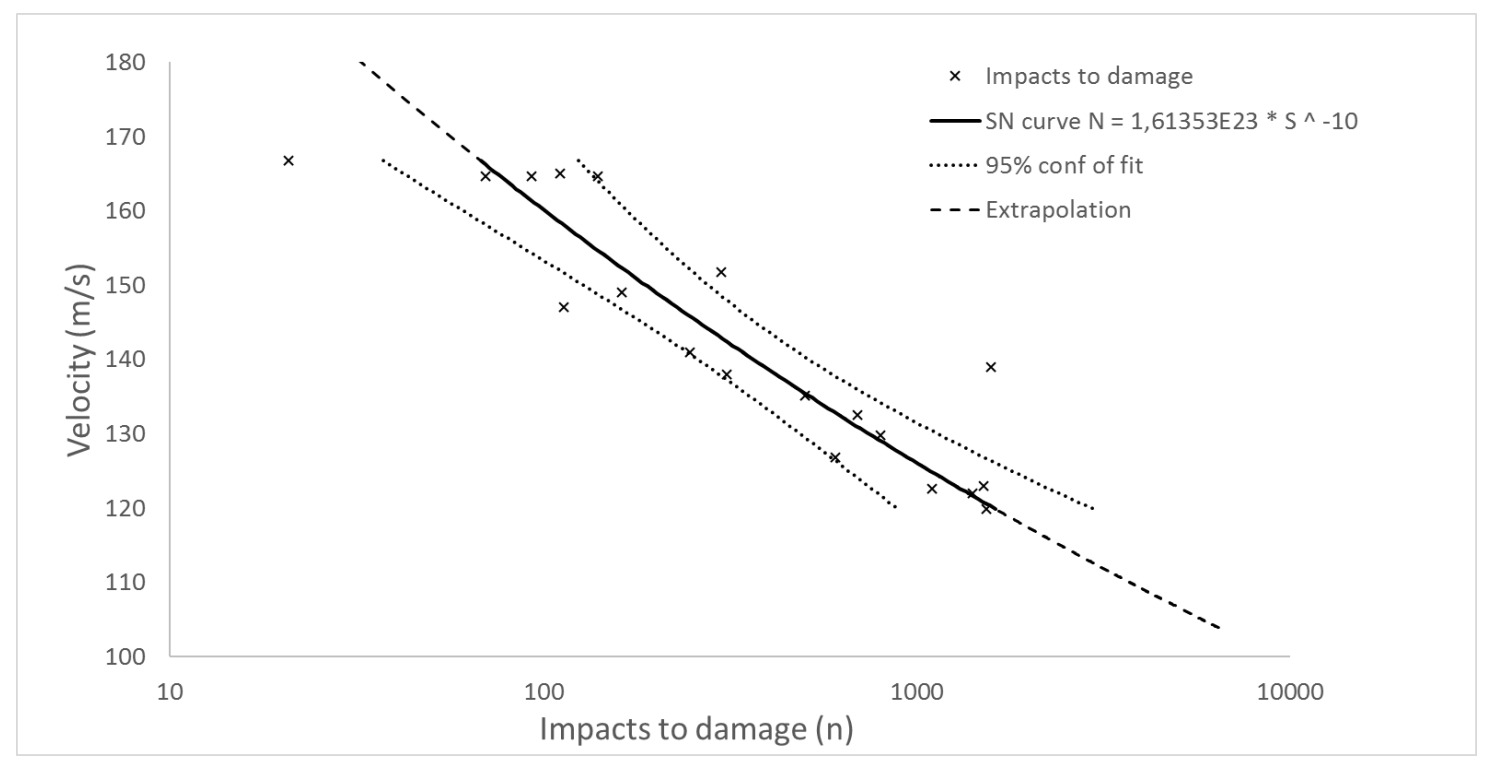

Figure 10. Semi-Log plot showing number of impacts to end of incubation at a set impact velocity fitted to a power curve.

\subsection{Experimental results from $A E$}

After running several SPIFT series with AE sensing, it became apparent that a significant change was occurring in the recorded $\mathrm{AE}$ signal for impact events at the immediate point where the coating was visibly damaged (see image $\mathrm{C}$ in Figure 9). An example of the $\mathrm{AE}$ feature read-outs generated during one of these test series is shown below.

Figure 11 shows a sharp change in average frequency of the AE hits recorded during the SPIFT series occurring at the point when the coating started to erode from the surface of the specimen. The group of hits from before this point (coating intact) is distinct from the group of hits recorded afterwards (coating eroded).

Figure 11 also shows that there is a change in amplitude of the AE hits recorded during the SPIFT series at the point when the coating is eroded from the surface of the specimen. In this case, the eroded coating results in multiple hits being recorded for each rubber ball impact, similar to the problem described in section 2.5. Ignoring these low amplitude secondary hits there is not a large change in amplitude following initial visible coating damage. 
There is, however, a very significant change in duration of the AE hits recorded during the SPIFT series at the point where the coating becomes visibly damaged.

A change in the energy of each AE hit recorded during the SPIFT series is observed at the point where the coating is visually seen to be damaged. What is also apparent from this feature is that a progression can be observed in the energy extracted from the AE hits as the impact series goes on. The energy content of the individual hits seems to be increasing up until the point where the coating is damaged when a step change (downwards) takes place. Following this, a similar progression of increasing energy for the individual AE hits is again observed.

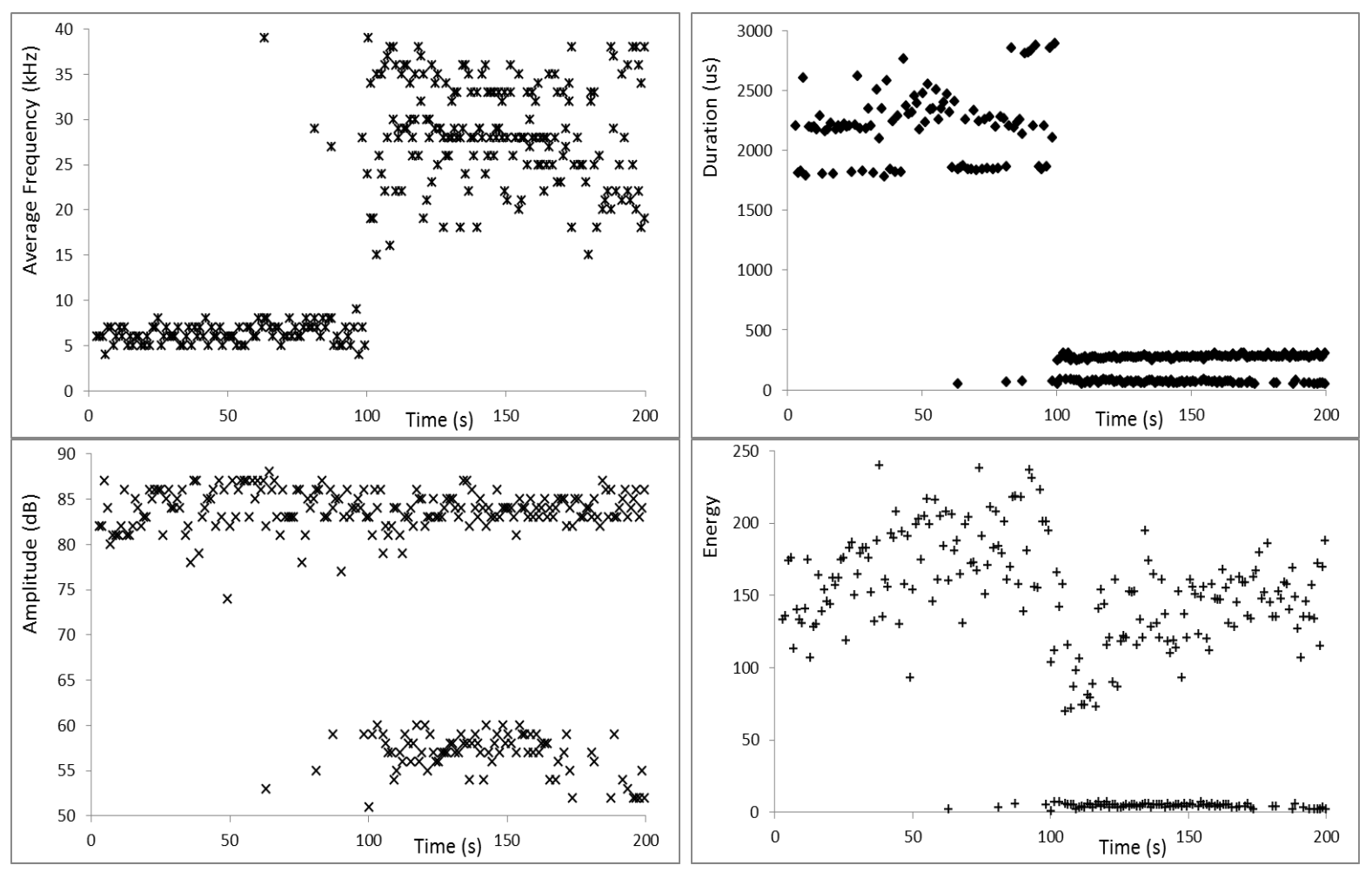

Figure 11. AE features during a typical SPIFT series at impact rate of $1 \mathrm{~Hz}$; the coating is eroded after $100 \mathrm{~s}$.

Summarising these changes in Table 3, we can see that when the coating is damaged, the average carrier frequency of the $\mathrm{AE}$ hit waveform increases, the maximum amplitude of the hits remains the same, the duration of each individual AE hit decreases sharply, and the total energy contained in the AE waveform goes down slightly.

It may seem counterintuitive that the energy recorded by the sensor on the reverse of the SPIFT specimen should decrease for each rubber ball impact once the coating is removed. But it is important to bear in mind that the energy feature of the $\mathrm{AE}$ waveform returns the energy content of the entire waveform detected but says nothing about the morphology of that waveform. The energy content of each detected AE waveform is related to the area above the threshold voltage and under the shape described by the waveform. Note that as soon as the coating is damaged, the duration of the waveforms detected is seen to be sharply reduced while the maximum amplitude remains the same. 
If we consider how much energy is being transferred into the laminate where the AE sensor is attached as a

Table 3. AE features average value change after coating erosion.

\begin{tabular}{|l|c|l|c|l|}
\hline AE waveform characteristic & \multicolumn{2}{|l|}{ hits prior to initial visible damage } & \multicolumn{2}{c|}{ hits after initial visible damage } \\
\hline & mean & St.dev. & Mean & St.dev. \\
\hline Av- Frequency $(\mathrm{kHz})$ & $\mathbf{7}$ & $71 \%$ & $\mathbf{2 5}$ & $16 \%$ \\
\hline Amplitude $(\mathrm{dB})$ & $\mathbf{8 3}$ & $7 \%$ & $\mathbf{8 4}$ & $2 \%$ \\
\hline Duration $(\mu \mathrm{s})$ & $\mathbf{2 1 2 3}$ & $24 \%$ & $\mathbf{2 7 7}$ & $5 \%$ \\
\hline Counts & $\mathbf{1 3}$ & $23 \%$ & $\mathbf{7}$ & $14 \%$ \\
\hline Energy & $\mathbf{1 6 7}$ & $27 \%$ & $\mathbf{1 3 5}$ & $21 \%$ \\
\hline
\end{tabular}

319 Table 4. Energy per unit time calculation.

\begin{tabular}{|c|c|c|c|}
\hline & Energy & Duration (ms) & Average energy per millisecond \\
\hline $\begin{array}{c}\text { Average AE hit when the } \\
\text { coating is intact }\end{array}$ & 167 & 2.123 & 79 \\
\hline $\begin{array}{c}\text { Average AE hit after initial } \\
\text { coating damage }\end{array}$ & 135 & 0.277 & 487 \\
\hline
\end{tabular}

The average energy per unit time for each rubber ball impact before the coating erosion is 79 units per ms, and after the coating is removed this goes up to 487 units per ms. The result is a significant increase $(\times 6)$ in the strain rate experienced by the bulk laminate following the erosion of the coating.

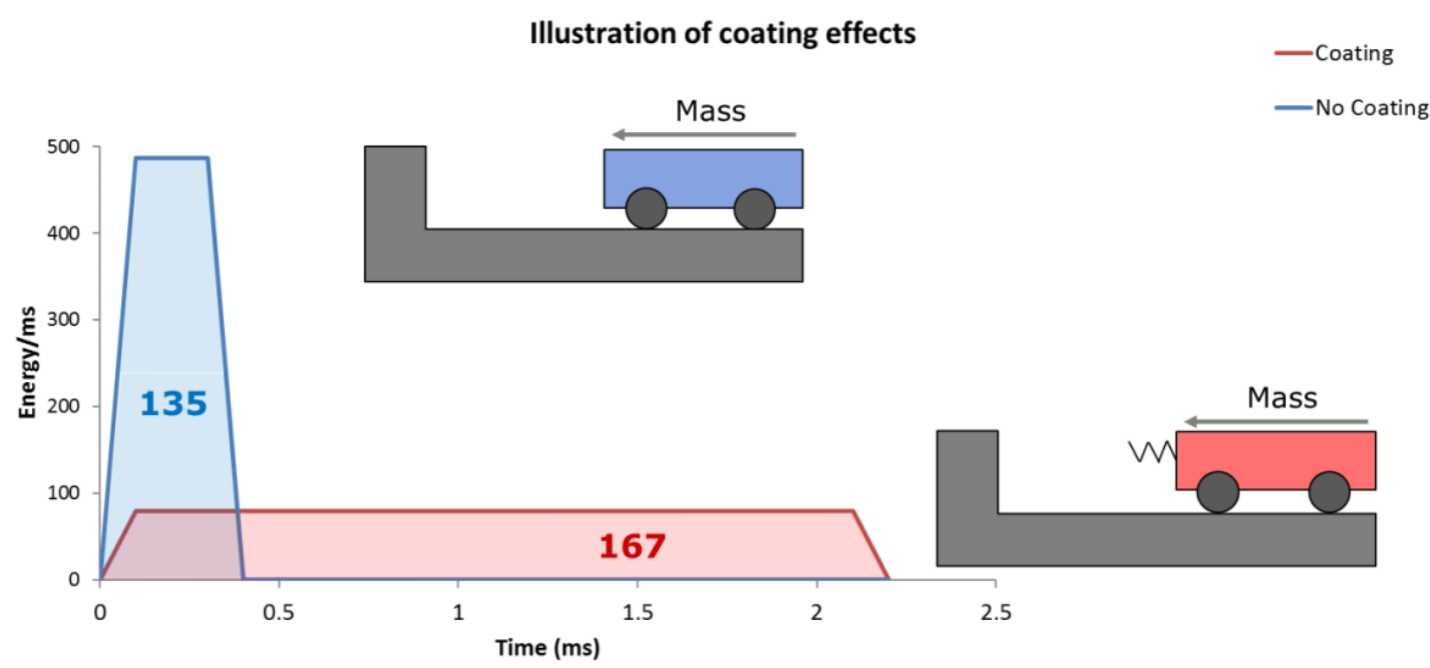

Figure 12. Coating effects illustration, comparison to crumple zone.

Illustrating the phenomena visually in Figure 12, we can see that although the hits on the specimen when the coating is intact contain slightly more absolute energy, this energy is distributed over a significantly longer period of time (over $2 \mathrm{~ms}$ ) at a rate of 79 units of energy per ms. Whereas the hits when the coating is removed record energy at a rate of 487 units of energy per ms for slightly less than $0.3 \mathrm{~ms}$ total duration. 
In this way, the coating on the laminate specimen acts like a crumple zone in a car subjected to impact. The energy transferred from a moving object striking a stationary object is the same, but the crumple zone design of the car ensures that this energy is distributed in time, thus saving the occupant from a potentially deadly deceleration.

From the initial measurements of AE on SPIFT test series, we can confirm that it is possible to detect changes in the recorded $\mathrm{AE}$ signal that corresponds to the damage condition of the coating and laminate. It appears to be possible to classify the types of $\mathrm{AE}$ waveforms from each impact to determine if the coating is functioning acceptably, if the coating is not performing as it should, and if the coating has been removed.

\subsection{Ultrasound}

Specimens were scanned both before and after they were exposed to impact damage.

In Figure 13 a specimen was scanned before impact test. Reflection of ultrasonic waves happens when there is a change in acoustic impedance Z. Using common values [9] for impedance $\mathrm{Z}$ of epoxy resins of between 1.7-2.4 $\left(\mathrm{MPa} \mathrm{s} \mathrm{m}^{-1}\right)$ and glass fibres 13.3-14.5 $\left(\mathrm{MPa} \mathrm{s} \mathrm{m}^{-1}\right)$, it is clear that there is a significant difference in impedance between the two materials. It is concluded in Figure 13 that the high signal area (yellow-magenta lines) is the reflected signal form the first layer of fibres $\left(45^{\circ}\right.$ oriented).

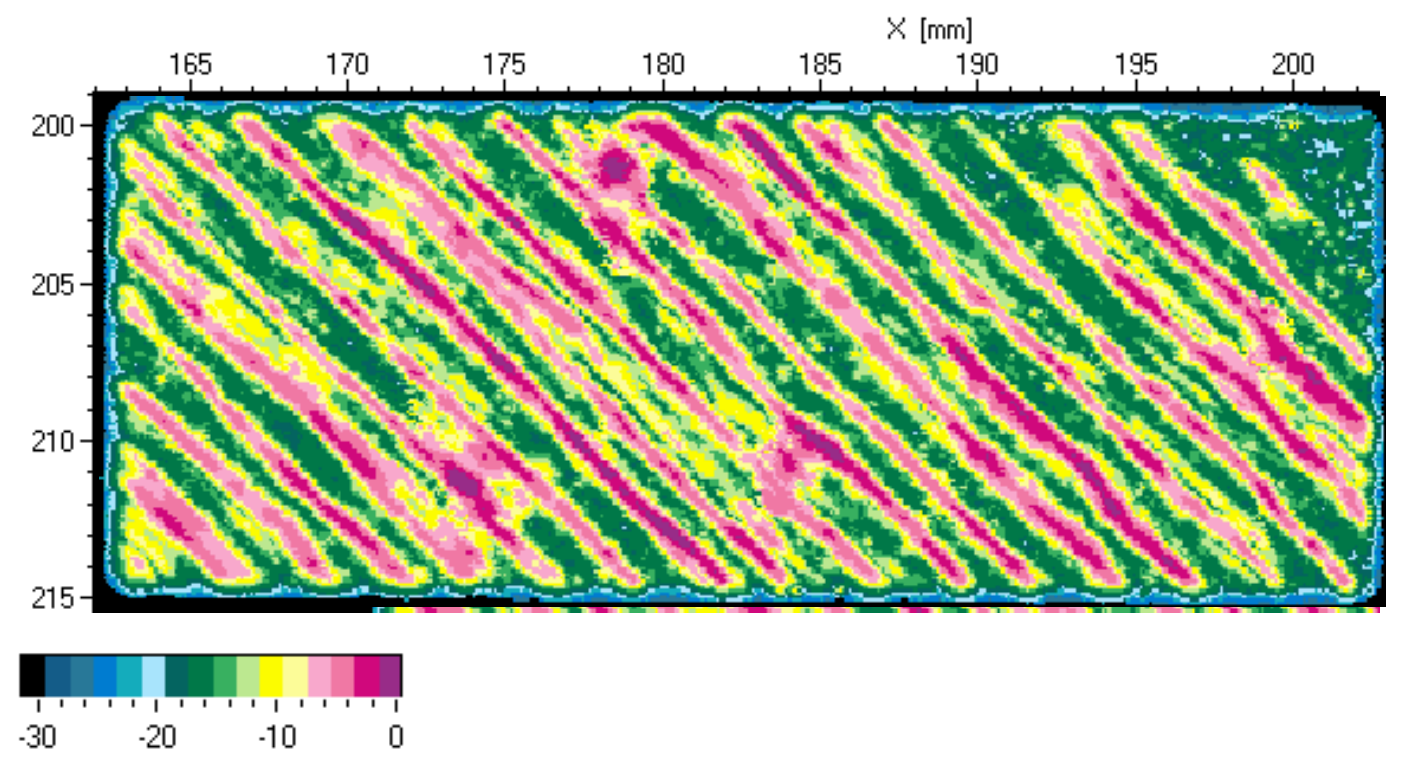

Figure 13. Ultrasonic scan from a specimen without impact damage (Specimen EMB-02). The $45^{\circ}$ rovings of the upper ply show as lines of low damping in the scan.

In Figure 14 the specimen was scanned after 140 impacts at $164 \mathrm{~m} \mathrm{~s}^{-1}$. The scan shows the impacted area.

Looking closer at Figure 14 there is a circular area of light blue signal with a diameter of roughly $3 \mathrm{~mm}$. Despite the sample having clear surface erosion damage, it is the area that shows circular crazing but no surface erosion that influences the ultrasound scan most. Many factors can lower the strength of the reflected signal but likely candidates are reflection or diffuse reflection of the incoming ultrasound signal as shown in Figure 7 in section 2.6. 


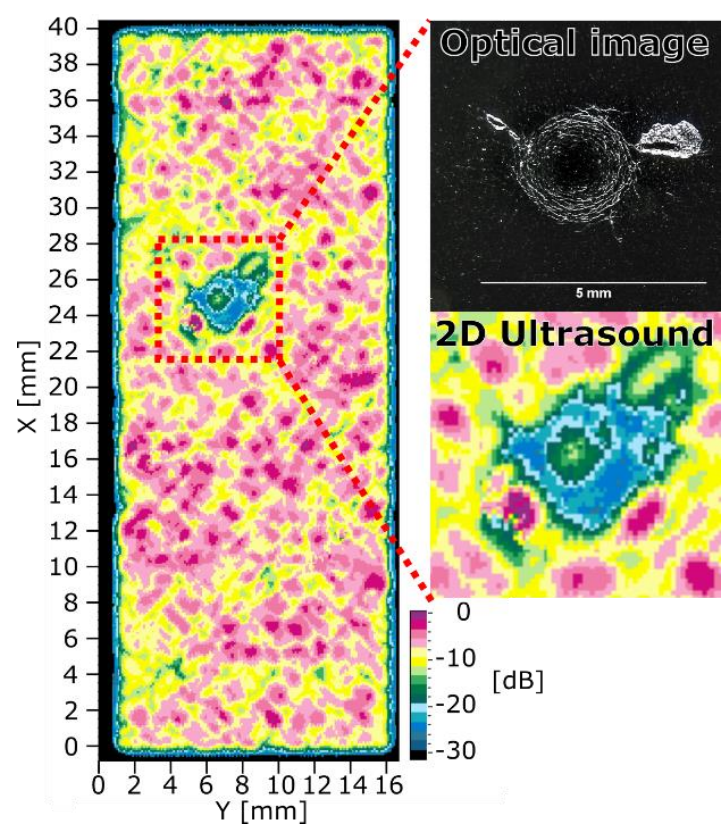

360 Figure 14. Scan from the specimen with impact damage from air gun overlaid with a visual image of the damaged sample. In the impacted area, there is a clear loss of signal compared to the surrounding area that shows the crosshatch pattern from the first and second glass fibre layers.

From the CT-scan in section 3.4 below, there are conical cracks can reduce the reflected signal. This reduced signal is likely a result of incoming ultrasound signal reflected away from the transducer due to the $45^{\circ}$ cracks in the impacted region. With a impedance of around $0.43 \cdot 10^{-3} \mathrm{MPa} \cdot \frac{\mathrm{s}}{\mathrm{m}}$ any air gap inside the conical cracks will result in a strongly reflected signal.

Using ultrasound scanning in this way provides a good nondestructive tool to quickly access the presence or extent of damage resulting from impact with typical scan times ranging between 5-10 min. This makes it an ideal first analysis tool before applying more expensive and time-consuming methods like CT-scanning.

By scanning laminates between impact series, the development of damage can be followed non-destructively. This applies not only to the SPIFT setup but, if correctly implemented, could also be adapted to the curved whirling arm RET specimens, either by just scanning the part of the leading edge parallel to the transducer, or by mounting the transducer to the specimens, capable of following the surface contour.

\section{$3 D$ X-ray tomography}

Damage mechanisms can be identified and followed using the 3D X-ray tomography. Figure 15 shows a 3D volume of the tested specimen. This scan is a superposition of three smaller scans (each $2 \mathrm{~mm}^{3}$ ). This procedure is called stitching and permits the study of a large volume with a small pixel resolution. The impact area is clearly observed and its diameter can be measured. It is found that several circular cracks were formed around the impact area.

First, the damage mechanisms appearing during single point impact fatigue need to be investigated in order to understand the failures. The observation indicates that this particular gelcoat includes large particle fillers that appear to be responsible for the crack initiation which occurred before visual damage of the partial coating removal. The fillers are acting as stress concentrating defects which facilitate the crack propagation within the 
material (Figure 15). The total diameter of the damage region is $2.3 \mathrm{~mm}$ to $3.0 \mathrm{~mm}$ for this specific gelcoat material.

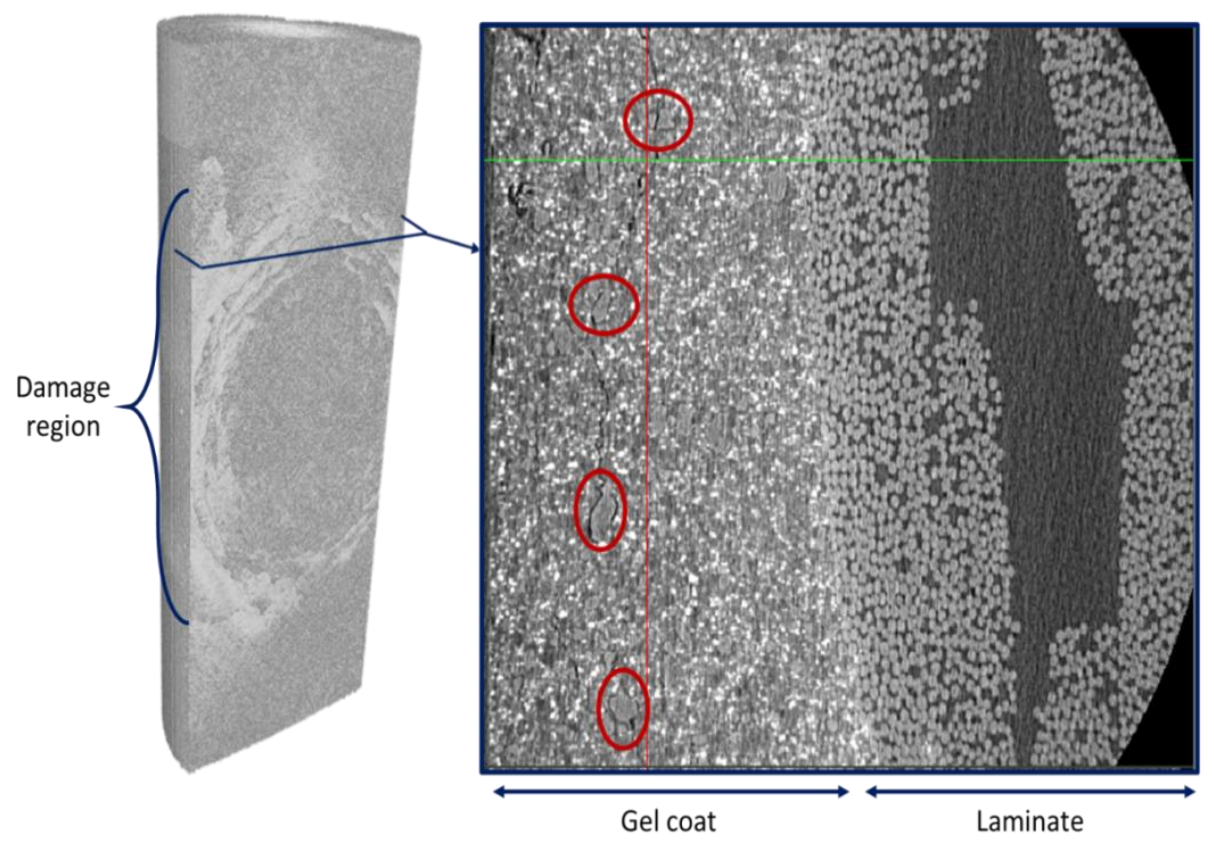

Figure 15. Damage mechanism in the gelcoat. Damaged region: $2.3-3.0 \mathrm{~mm}$.

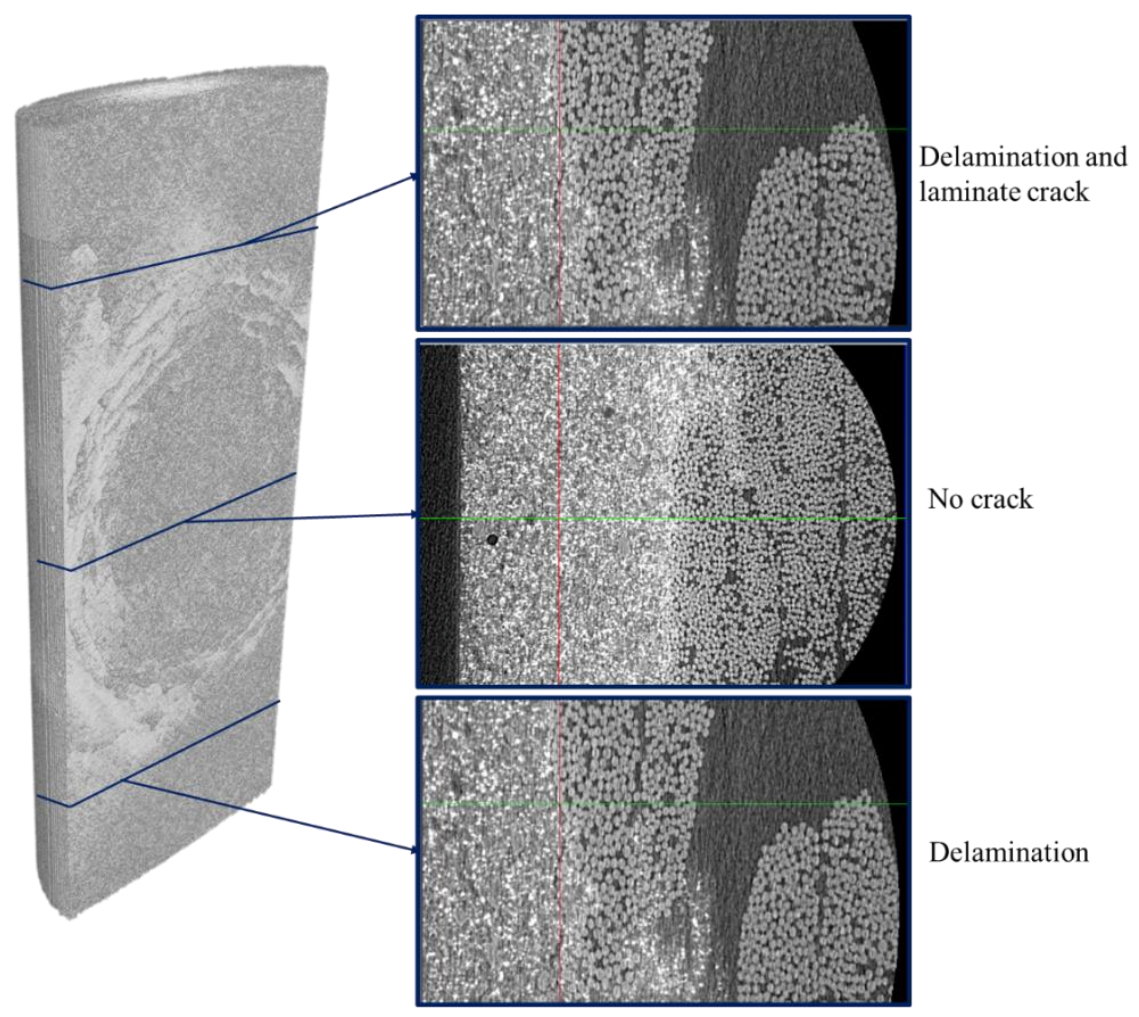

417 Figure 16. Damage localisation in the impact region. 
A further study of the entire impact zone reveals an interesting phenomenon. At the centre of the impact zone, no cracks are identified in the gelcoat. Near the centre of the impact zone, there are no or only a few cracks. Moving from the centre towards the rim, the first cracks appear at around $75 \%$ of the outer diameter, and they increase in density near the edge of the zone. The cracks are ring-shaped at the surface of the gelcoat, and they extend into the gelcoat at an angle of $45^{\circ}$ thus forming cone shaped crack surfaces. Outside the impact, cracks are running closer to the interface; delamination can be identified in this region (Figure 16). The conic shape of the impact cracks through the gelcoat thickness is to be expected and has been observed in other materials subjected to impact.
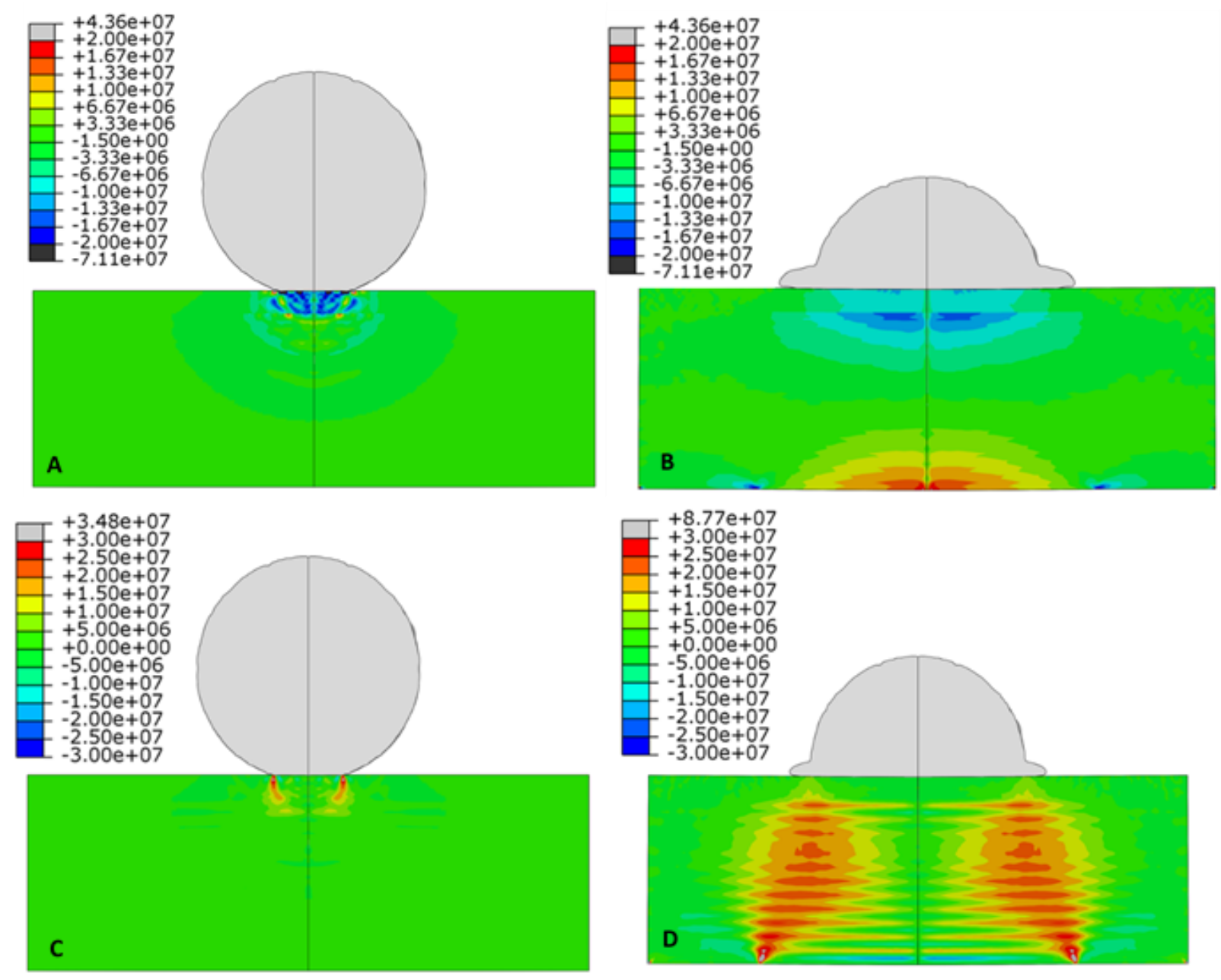

Figure 17. Stresses in the laminate under nitrile ball impact. A and B: $\sigma_{11}$ stress at the transient (5 $\left.\mu \mathrm{s}\right)$, and quasi static stage $(21 \mu \mathrm{s})$. C and D: $\sigma_{13}$ stress at the transient and quasi static stage.

The results of the simulations in tensile stress are shown in Figure 17. It can be seen that while at the beginning (transient stage of the impact), the highest stress is localised under the contact surface, at the quasi-static stage the high stress region forms in the depth under the surface. Similarly, rather high shear stresses form at the angle $30^{\circ}$ to $45^{\circ}$ under the contact surface. 
Further, the comparison between the laminate deformation under rubber ball and water droplet impacts have been carried out (at the loading rate $80 \mathrm{~m} \mathrm{~s}^{-1}$ ). It was observed that the shear stresses in the laminate are $70 \%$ higher under the water drop (17 vs $10 \mathrm{MPa}$ ) than under the rubber ball.

The results of simulations have been compared with experiments. CT scanning of the specimens revealed cracks in gelcoat at 30 to $45^{\circ}$ relative to the surface as shown in Figure 18. The cracks observed in the scan are in agreement with the FEM simulation the shear stresses is also presented.
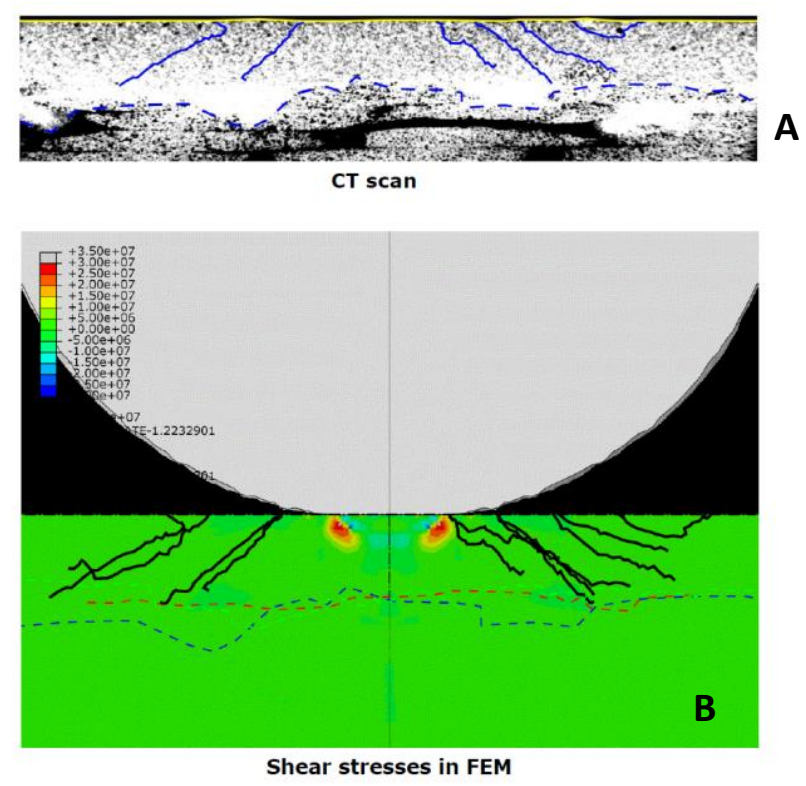

Figure 18. Comparison of the cracks observed in CT scan and the shear stress distribution as well as delamination between the laminates and coating represented by the dashed lines. The blue lines in Figure A are drawn on top of the observed cracks and superposed in B together with lines coming from two more tested samples.

4.

\section{Conclusion}

Experimental and theoretical assessments for damages caused by repeated high speed impacts were presented. SPIFT demonstrated repeated impacts on a coated laminate with rubber balls, damaging the coating and the laminate. In-situ video observation and AE monitoring and ex-situ characterisations by ultrasonic scanning and X-ray CT-scan were carried out.

In-situ video data enabled determination of the incubation time and plotting of SN curves in terms of coating removal at an impact speed ranging between 123 and $165 \mathrm{~m} \mathrm{~s}^{-1}$. It is demonstrated that the incubation time obtained by the video observation showed correlation to the change of $\mathrm{AE}$ signal recorded in the in-situ $\mathrm{AE}$ data. The ex-situ characterisations by ultrasound and CT scans revealed that damage could be induced in the coating and the laminate before the visible damage started. In particular, the ex-situ CT scans were capable of identifying conical cracks in the coating resulting from the repeated impacts. Additional repeated impacts extended the cracks into the laminate. An FEM model indicated intense shear-stress components, showing a good agreement with the coating cracks observed with the CT-scans. 
465

466

467

468

469

470

471

472

473

474

475

476

477

478

479

480

481

482

483

484

485

486

487

488

489

490

491

492

493

494

495

496

497

498

499

500

501

The techniques presented can be used for studying high speed impact damages, in particular, leading edge erosion of wind turbine blades. The in-situ methods demonstrated immediate detection of changes and damages while the ex-situ methods as well as the modelling can provide dedicated analysis. It is therefore suggested that combination of these techniques will enable efficient investigation of the complicated high speed impact damage processes that can be seen in reality, for example, in the leading edge erosion of wind turbine blades.

\section{Acknowledgements}

The authors are grateful to Lars Lorentzen for developing SPIFT, Erik Vogeley for operation of the X-ray CTscan, and Christian H. Madsen and Jonas K. Heininge for fabricating laminate specimens. This research was partly supported by EUDP project 'Rain erosion tester for accelerated test of wind turbine blades', Case no.: 64015-0045 (EUDP) and the Innovation Fund Denmark being part of the Fast-Track consortium (515200002B). This research was conducted using mechanical testing equipment from Villum Center for Advanced Structural and Material Testing (CASMAT), award reference 00007293 from Villum Fonden.

\section{References}

1. Progress and recent trends of wind energy technology. M.R Islam, S. Mekhilef, R. Saidur. s.I. : Renewable Sustainable Energy Rev., 2013, Vol. 21. 456-468.

2. The evolution of wind turbine design analysis-a twenty year progress review. D.C Quarton s.I. : Wind Energy, 1998, Vol. 1. 5-24.

3. Why offshore wind energy? M.D Esteban, J. Diez, J. S. Lopez, V. Negro. s.I. : Renewable Energy, 2011, Vol. 36. 444-450.

4. The wind energy (r)evolution: A short review of a long history. J.K Kaldellis, D. Zafirakis. s.I. : Renewable Energy, 2011, Vol. 36. 1887-1901.

5. Wind Turbines: How Big can they Get? G. Marsh. s.I. : Refocus, 2005, Vol. 6. 22-28.

6. C. Red, Composites Technology. s.l. : Composites World, Web Publisher, 2008.

7. On erosion issues associated with the leading edge of wind turbine blades. M.H Keegan, D.H Nash, M.M Stack. s.I. : J. Phys. D Appl. Phys., 2013, Vol. 46. 383001.

8. Computational analysis of wind turbine blade erosion. A.Castorrini, A. Corsini, F. Rispoli, P. Venturini, K. Takizawa, T.E Tezduyar. s.I. : Computers and Fluids, 2016, Vol. 141 175-183.

9. Leading Edge Erosion of Coated Wind Turbine Blades: Review of Coating Life Models. H.M Slot, E.R.M. Gelinck, C. Rentrop, E. Van der Heide. s.I. : Renewable Energy, 2015, Vol. 80. 837-848.

10. Rain erosion of wind turbine blade coatings using discrete water jets: Effects of water cushioning, substrate geometry, impact distance and coating properties. S. Zhang, K. Dam-Johansen, P.L. Bernad Jr, S. Kiil. s.l. : Wear, 2015, Vols. 328-329 140-148.

11. 2012, ASTM. ASTM G73-Standard Test Method for Liquid Impingement Erosion Using Rotating Apparatus. 2012. 
12. Impact fatigue damage of GFRP materials due to repeated raindrop collisions. G. Prayogo, H. Homma, 503 T. P. Soemardi, A.S. Danardono. s.I. : Trans . Indian Inst. Metals, 2011, Vols. 64 (4-5). 501-506.

13. Cumulative fatigue damage and life prediction theories: a survey of the state ofthe art for homogeneous materials. A. Fatemi, L. Yang. s.I. : Int. J. Fatigue, 1998, Vol. 20(1). 9-34.

14. Cumulative Damage in Fatigue. K. Reifsnider, S. Case, J. Duthoit. s.I. : Composites Sci. Technol., 2000, Vol. 60. 2539-2546.

15. Multiple Liquid Impacts on Polymeric Matrix Composites Reinforced with Carbon Nanotubes. $\mathbf{0}$. Gohordani, D.M. Williamson, D.W. Hammond. s.I. : Wear, 2012, Vols. 294-295. 336-346.

16. Multiple Impact Jet Apparatus (MIJA): Application To Rain Erosion Studies. P.N.H. Davies, J.E. Field. s.I. : Proc. SPIE 1112, Window and Dome Technologies and Materials, 1989. Vol. 1112. doi: 10.1117/12.960790.

17. Estimation of fatigue curves for design of composite laminates. K.0. Ronold, A.T. Echtermeyer. s.I. : Composites A, 1996, Vol. 27. 485-491.

514 18. A review of wind energy technologies. G.M Herbert, S. Iniyan, E. Sreevalsan, S. Rajapandian. s.I. : 515 Renewable Sustainable Energy, 2007. Vol. 11 (6) 1117-1145.

\section{19. [Online]}

517 http://www.physicalacoustics.com/content/literature/multichannel_systems/Express_8_Product_Bulletin. 518 pdf.

519 20. [Online] http://www.physicalacoustics.com/content/literature/sensors/Model_Nano30.pdf.

520 21. Leading edge erosion of coated wind turbine blades: Review of coating. H.M. Slot, E.R.M. Gelinck, C. 521 Rentrop, E. van der Heide. s.I. : Renewable Energy, 2015. Vol. 80. 837-848. 